\title{
Empleo de prebióticos y probióticos en el manejo de la ansiedad
}

\author{
Violeta Cepeda-Vidal', Alicia Mondragón-Portocarrero², Alexandre Lamas², José Manuel Mirandaª \\ Alberto Cepeda ${ }^{1,3}$ \\ 1. Farmacéuticos comunitarios. 2. Investigadores postdoctorales. 3. Profesor Área Nutrición y Bromatología (Universidad de Santiago de Compostela).
}

\section{PALABRAS CLAVE}

Ansiedad, Bifidobacterium, Lactobacillus, polisacáridos, microbiota, probiótico

\section{ABREVIATURAS}

AGCC: Ácidos grasos de cadena corta $\beta$-GOS: $\beta$-galactosacáridos CCK: Colecistoquinina

ECCs: Células entero-endocrinas

FOS: Fructooligosacáridos

GABA: Ácido g-animobutírio

GLP-1: Péptido de glucagon 1

GOS: Galactooligosacáridos

LAB: Bacterias ácido-lácticas

LPS: Lipopolisacárido

MI: Microbiota intestinal

NPY: Neuropéptido Y

miRNA: microARN

ob/ob: predisposición genética a la obesidad

POMC: neuronas pro-

opiomelanocortina

PYY: Péptido YY

SNA: Sistema nervioso autónomo

SNC: Sistema nervioso central

Spp.: Especie

\section{KEYWORDS}

Anxiety, Bifidobacterium, Lactobacillus, polysaccharides, microbiota, probiotic

\section{RESUMEN}

El objetivo del presente trabajo ha sido realizar una recopilación de la última evidencia científica acerca de las vías de comunicación entre el sistema nervioso y el intestino, así como la influencia de la microbiota intestinal en la ansiedad. Posteriormente se ha recopilado información acerca de los ensayos preclínicos y clínicos con prebióticos y probióticos para el manejo de la ansiedad y se ha discutido su eficacia y seguridad. También se mencionan las ventajas de la administración de prebióticos y probióticos mediante presentaciones farmacéuticas con respecto a la suplementación por vía alimentaria.

Para la realización del mismo se ha realizado una búsqueda en bases de datos científicas, empleando sólo artículos publicados en revistas de prestigio internacional y priorizando los artículos publicados en los últimos años.

La microbiota intestinal y el sistema nervioso central se encuentran conectados mediante varios mecanismos, que de manera colectiva se denominan "eje intestino-cerebro". Según evidencias recientes, la vía de comunicación más importante entre ambos órganos la constituye el nervio vago.

Los pacientes suelen ser muy reticentes a cambiar de manera importante sus hábitos alimentarios. Así, la administración de prebióticos y probióticos mediante presentaciones farmacéuticas puede facilitar este proceso, controlando mejor la dosis administrada y disminuyendo las tasas de abandono precoz de los tratamientos. Si bien no en todos los casos los prebióticos y probióticos han mostrado efectos positivos, en ninguno se han demostrado efectos adversos en respuesta al empleo de los mismos, por lo que se puede concluir que su administración es segura.

Use of prebiotics and probiotics for the management of anxiety

\section{ABSTRACT}

The aim of this work has been to compile the latest scientific evidence about the communication pathways between the nervous system and the intestine, as well as the influence of the intestinal microbiota on anxiety. Subsequently, information has been collected about preclinical and clinical trials with prebiotics and probiotics for anxiety management and their efficacy and safety has been discussed. The advantages of administering prebiotics and probiotics through pharmaceutical presentations with respect to foods are also mentioned.

For this purpose, a search has been carried out in scientific databases, using only articles published in prestigious international journals and prioritizing the articles published in recent years.

The intestinal microbiota and the central nervous system are connected by various mechanisms, which are collectively called the "intestine-brain axis". According to recent evidence, the most important means of communication between the two organs is the vagus nerve.

Patients are often very reluctant to change their eating habits significantly. Thus, the administration of prebiotics and probiotics by means of pharmaceutical presentations can facilitate this process, better controlling the dose administered and decreasing the rates of early abandonment of treatments. Although in all cases prebiotics and probiotics have not shown positive effects, in none have shown adverse effects in response to the use of them, so it can be concluded that their administration is safe. 


\section{Introducción}

Hasta hace apenas dos décadas, la influencia de la microbiota intestinal (MI) en la salud humana se subestimaba, en gran parte debido a la imposibilidad de detectar gran cantidad de especies bacterianas mediante las metodologías microbiológicas clásicas (1). En concreto, fue a partir del año 2010 a raíz del trabajo De Filippo y col (2) en el que se demostraban las diferencias en la microbiota intestinal de niños europeos y de África rural cuando la investigación en este campo se incrementó exponencialmente. De la gran multitud de microorganismos que habitan en el cuerpo humano, sólo una minoría de bacterias podían ser cultivadas mediante dichos métodos, por lo que existía un gran desconocimiento sobre la actividad, cantidad y calidad de las bacterias que habitaban en el cuerpo (3). Por ello, con el desarrollo de nuevas metodologías en estos últimos años, actualmente conocemos que existen aproximadamente diez veces más microorganismos dentro del tracto gastrointestinal humano que células somáticas hay en el cuerpo $(3,4)$.

Tanto la MI como los cambios en la misma juegan un papel decisivo en gran cantidad de patologías, como son la enteritis, el cáncer, la diabetes, la obesidad, los trastornos digestivos, etc. (5-7). Entre sus múltiples aplicaciones, se ha descubierto que algunos componentes de la MI tienen capacidad para influir en nuestro estado de ánimo y modificar nuestro comportamiento en algunos aspectos como la respuesta a la ansiedad (8).

La ansiedad puede definirse como una reacción de nuestro organismo a una situación persistente de estrés (9). Situaciones de estrés recurrentes provocan una situación de ansiedad sostenida en el tiempo, que si no es tratada de un modo adecuado podría desencadenar enfermedades mentales como la depresión (10). Una de las consecuencias de la ansiedad continuada y de las enfermedades mentales que de ella derivan es que causan un incremento de los niveles de glucocorticoides que se mantienen en el tiempo produciendo una activación del eje hipotalámico-hipófisis-suprarrenal y cambios en la estructura del hipocampo, conduciendo posteriormente a alteraciones en la neurogénesis, en la morfología neuronal e incluso son capaces de producir la muerte celular de las neuronas (11).
Algunos autores afirman que los tratamientos medicamentosos existentes, no son totalmente efectivos para tratar trastornos mentales como son la ansiedad y la depresión (9, 11). La mayor parte de los tratamientos se basan en fármacos antidepresivos, como los inhibidores selectivos de la recaptación de serotonina $(9,12)$. No obstante, este grupo de fármacos tienen efectos secundarios perjudiciales como la reducción del apetito sexual, el aumento de peso y trastornos en el sueño (11). De este modo, los pacientes que están en tratamiento con ansiolíticos y antidepresivos experimentan sintomas residuales en un 30\%-60\%, y entre un $10 \%-20 \%$ experimentan recaídas frecuentes (9), lo que en no pocos casos provoca el abandono prematuro del tratamiento (11). Otra consecuencia de la ansiedad es que a menudo se manifiesta mediante una activación persistente del apetito (12).

Por este motivo, una de las estrategias terapéuticas que se han introducido en los últimos años consiste en influir en los síntomas de la ansiedad a través de los factores ambientales que influyen en esta (13). A este respecto, la MI se ha planteado como una importante vía de intervención (14).

\section{Mecanismos de regulación de la ansiedad}

La sintomatología clínica de la ansiedad responde a una excitación del sistema nervioso autónomo (SNA), especialmente el sistema simpático. A partir de esta excitación se producen como consecuencia diversos sintomas cardiorrespiratorios, gastrointestinales, etc. En pacientes con trastornos de ansiedad existe una respuesta anormal y excesiva del SNA frente a diferentes estímulos y en situaciones de reposo comparados con los controles.

La regulación de la ansiedad es muy compleja e intervienen en ella numerosos agentes, como son el sistema noradrenérgico, el sistema serotoninérgico, el ácido g-aminobutírico (GABA), el eje hipotalámico-hipofisario-adrenal y una importante cantidad de neurotransmisores como el neuropéptido Y (NPY) y la colecistoquinina (CCK) $(15,16)$.

Entre todos estos factores y sistemas tan complejos también se ha demostrado que la MI también es capaz de influir en numerosas funciones cerebrales, entre las que se encuentra la regulación de la ansiedad (3). Existen varias vías de comunicación entre el intestino y el cerebro, como por ejemplo: aferentes vagales; hormonas intestinales; citoquinas, y comunicación mediante exosomas, que son membranas lipopolisacáridas que no tienen por qué circular exclusivamente por las rutas anteriormente mencionadas pero que pueden alcanzar cualquier localización dentro del organismo $(17,18)$. Recientemente, se ha podido observar que las lesiones cerebrales también son capaces de afectar potencialmente la composición de la MI y causar un estado de disbiosis, entendiéndolo como todo estado de la MI que se aleje del estado de equilibrio que se considera saludable (1).

La MI ha demostrado desempeñar un papel clave en la activación de las microglías (19), por lo que su manipulación, especialmente a través de bacterias que son capaces de fermentar sustratos y, de este modo, producir en el intestino grueso ácidos grasos de cadena corta (AGCC), podría modular la activación neuro-inmune (20).

\section{Importancia de la microbiota intestinal}

La composición de la MI en un ser humano adulto depende del efecto que van teniendo sobre ella numerosos hechos influyentes en la vida de una persona (4). Entre ellos, tienen una gran influencia si el nacimiento fue natural o mediante cesárea, el tipo de alimentación durante la lactancia, las condiciones higiénicas en las que viva, el uso de antibióticos, el ejercicio o la edad, entre otros (1). No obstante, el principal agente modulador de dicha MI es la dieta, la cual puede variar su composición de manera rápida (21). Una dieta elevada en grasas, típica del mundo occidental, retroalimenta a la obesidad, en parte por la modificación de la MI y la generación de un deterioro de la conducta, aumentando el comportamiento ansioso (22). Por el contrario, una dieta alta en fibra contribuye a reducir el índice de masa corporal y ejerce una acción indirecta sobre el sistema inmunitario, modulando el desarrollo de la MI, pues favorece la colonización de géneros bacterianos beneficiosos como Bifidobacterium spp. (4, 23). Por su 
parte, una dieta rica en proteínas y baja en fibra resulta en un incremento de ácidos grasos de cadena larga y otros metabolitos tóxicos como el amonio, indol, fenol y derivados (1). También influyen de una manera muy importante algunos tipos de micronutrientes, aditivos y contaminantes que pueden estar incluidos en los alimentos (1).

La disbiosis causada por una dieta con alto contenido en grasas también puede dar lugar a una MI disbiótica proinflamatoria, la cual produce gran cantidad de mediadores proinflamatorios como el lipopolisacárido (LPS) (24). Estos metabolitos generados por la MI proinflamatoria pueden modificar tanto la integridad como la permeabilidad de los enterocitos, desencadenando la liberación de citoquinas proinflamatorias como factor de necrosis tumoral $\alpha$ y la interleukina- $1 \beta$ (17). Estas citoquinas, a su vez, son capaces de alterar la integridad de las uniones entre las células epiteliales, modificando así la permeabilidad del epitelio colónico (24). Por ello, uno de los factores más importantes para la prevención de los trastornos metabólicos, es prevenir un incremento en la permeabilidad del intestino grueso.

\section{El eje intestino-cerebro}

La MI y el sistema nervioso central (SNC) están comunicados mediante un conjunto de vías diferentes de señalización, que se denominan de manera colectiva como "eje intestino-cerebro" (25). Este eje es un sistema bidireccional, en el que participan conexiones neurológicas directas, señales endocrinas y factores inmunológicos (26). Mediante este eje, la MI transmite al SNC información específica, en función de los alimentos y nutrientes que pasan a través del tracto gastrointestinal. Una vez el SNC recibe esta información, elabora una respuesta sistémica (13). De este modo, cuando se produce un estado de disbiosis, los mensajes que van desde el intestino al cerebro transmiten señales que causan estrés proinflamatorio (19), un incremento del estrés oxidativo, un desequilibrio en la homeostasis energética y un incremento en la degeneración celular (27).

Muchos autores defienden que la disbiosis contribuye al desarrollo de patologías neurológicas como la depresión, la ansiedad y la neurodegeneración $(19,25)$. Se ha llegado a la conclusión de que la MI y el cerebro están comunicados por cuatro tipos de conexión: autónoma, neuroendocrina, entérica e inmunológica (25).

A pesar de los múltiples mecanismos de comunicación a través de los cuales podrian interrelacionarse el intestino y el SNC, en los últimos años se ha postulado que la vía prioritaria de comunicación entre ambos la constituye el nervio vago (25). Mediante el nervio vago se transmiten la información referente a la cantidad y calidad de los nutrientes a los centros responsables de regular el comportamiento alimentario, que se encuentran principalmente localizados en el núcleo del tracto solitario, situado en el tronco encefálico $(28,29)$. Mediante este nervio uno de los mediadores más influyentes es el LPS, que tiene el potencial de incluso llegar a modificar la sensibilidad de las neuronas vagales (29).

Algunas de las bacterias comensales del tracto gastrointestinal pueden metabolizar diferentes tipos de nutrientes para producir, a partir de ellos, neurotransmisores como son la serotonina, el GABA, la dopamina y la noradrenalina, los cuales posteriormente pueden activar los terminales vagales (30).

La presencia de nutrientes en el tracto gastrointestinal inicia una cascada de procesos fisiológicos que informan al cerebro del estado nutricional de la persona. El nervio vago se activa de manera parcial con la secreción de ciertas hormonas como el péptido YY, péptido de glucagón 1 (GLP-1) y CCK, secretados por las células entero-endocrinas (ECCs) del intestino. Estos péptidos actúan en los receptores localizados en las fibras aferentes vagales, de manera que la información se transmite al nervio vago (31). Las ECCs están localizadas en contacto directo con el lumen y debido a esta proximidad, las bacterias comensales pueden interactuar directamente con ellas y, por tanto, modular la secreción de estos péptidos y otros metabolitos, los cuales se absorben y pasan al sistema circulatorio por transporte paracelular cuando la barrera epitelial está inflamada (32).

Por su parte, el sistema inmune también ejerce un papel importante. Está involucrado manteniendo la homeostasis entre la MI y el propio intestino, y actúa como un intermediario entre la MI y el SNC (2). Dicha MI libera ciertas moléculas que pueden ser promotores de la respuesta inmunita- ria inmediata. Cuando la integridad de la barrera epitelial intestinal se ve comprometida, las bacterias gram-negativas producen LPS, el cual puede llegar al sistema circulatorio y activar la respuesta inmunitaria por parte del organismo humano, hecho que favorece el desarrollo de la ansiedad (31).

Otra vía de comunicación entre la MI y el SNC, menos conocida, es la que tiene lugar a través de los microRNAs (miRNAs). Los miRNAs son un nuevo grupo de pequeños RNAs (aproximadamente 22 nucleótidos) no codificadores que han surgido como importantes reguladores de la expresión de ARN mensajero. En concreto, parecen tener gran influencia en la regulación de la leptina. La deficiencia o disfunción de la leptina al principio de la vida podría llevar a cambios profundos en la expresión génica, incluyendo patrones de expresión de ARN en los núcleos hipotalámicos.

Una primera aproximación para comprender el efecto de la leptina en los perfiles de miRNAs fue realizada por Benoit y col. $(33,34)$. Estos investigadores bloquearon la sintesis endógena de leptina en ratones recién nacidos y observaron los perfiles de miRNA en el hipotálamo de los ratones. Los resultados obtenidos mostraron que 34 miRNAs estaban regulados positivamente y 4 miRNAs estaban regulados negativamente. De los miRNAs que estaban positivamente regulados destacaron miR-10a, miR-200a, rno-miR-409-5p y miR125a. Otro estudio evaluó los perfiles de miRNAs del hipotálamo en situación de balance energético negativo (35), mostrando que un conjunto de miRNAs tuvieron patrones de expresión en el hipotálamo alterados por dieta de restricción calórica y/o una dieta alta en grasas (35).

La leptina modula la actividad de las neuronas pro-opiomelanocortina (POMC) que son reguladoras del equilibrio energético. Se ha visto que la interrupción del gen POMC causa hiperfagia y obesidad. En un estudio realizado por Derghal y col. (36) observaron que los miRNA-383, miRNA-384-3p, y miRNA-488 están aumentados en el hipotálamo de ratones ob/ob deficientes de leptina. Otro miRNA que ha demostrado estar relacionado con la ingesta de alimento es el miRNA-33, un miRNA relacionado con el metabolismo de los lípidos y la señalización de la insulina (37). 


\section{Microbiota y ansiedad}

A pesar de los muchos estudios que se han realizado recientemente en animales que describen la asociación entre la MI y la ansiedad, esta relación no ha sido muy investigada en humanos. Algunos trabajos, como el realizado por Stevens y col. (38), han encontrado en pacientes con ansiedad prolongada una MI con una menor diversidad de lo habitual, asi como una mayor proporción de bacterias pertenecientes al género $\mathrm{Bac}$ teroides. Kim y La Serre (17) también encontraron que la exposición crónica a situaciones de estrés da lugar a desregulaciones de la MI, incluyendo la bajada de niveles de Firmicutes, Bacteroidetes, y Lactobacillus spp., así como un incremento en los niveles de Oscillibacter spp. Adicionalmente, se ha realizado el experimento de trasplante de la MI de pacientes con depresión a ratas y se observó en éstas un cambio en la MI y el desarrollo de comportamientos propios de crisis de ansiedad (39). Esto abre la puerta a la posibilidad de que a través de la MI, se pueda intervenir en la prevención o en la mejora de los síntomas de la ansiedad. Generalmente, cuando se trata de modificar la MI no se intenta modificar totalmente, sino que se trata de modular el crecimiento de especies bacterianas beneficiosas mediante prebióticos o inocular alguna nueva especie, generalmente considerada probiótico. Los prebióticos se definen como ingredientes no digeribles que mediante su fermentación favorecen de modo especifico el crecimiento de un grupo de microorganismos beneficiosos para la salud (4). Los probióticos son agentes microbianos (no siempre bacterias) que pueden definirse como microorganismos vivos que ejercen efectos sobre la salud del huésped si se consumen en cantidades suficientes (4). Dentro de los probióticos, los Lactobacillus spp. y los Bifidobacterium spp. son los géneros bacterianos más habitualmente empleados, con un largo historial de uso seguro y beneficios para la salud humana demostrados, como el refuerzo de la inmunidad y la mejora de la función intestinal (4). Recientemente, se ha demostrado que estos géneros bacterianos también pueden tener beneficios en otras localizaciones del cuerpo humano, como es el SNC (40). Este hecho es consecuente con que la composición de la MI desempeña un papel clave en la predisposición a muchos trastornos mentales, incluido el trastorno depresivo mayor (41), el trastorno bipolar (42) y la ansiedad (38). Adicionalmente, algunos de los microorganismos que integran la MI también son potencialmente modificables por medio de los fármacos inhibidores selectivos de la recaptación de serotonina que ingiere el paciente (8).

Por todo ello, la MI puede ser una potencial diana terapéutica y preventiva para tratar la ansiedad, así como otros trastornos propios del SNC. Esta estrategia puede llevarse a cabo mediante dos maneras. La primera sería evitando y/o limitando los efectos de los agentes bacterianos que puedan alterar negativamente la permeabilidad intestinal. La segunda vía sería potenciando la presencia de aquellas especies bacterianas que tengan un efecto positivo sobre los mecanismos ya mencionados. Esto puede lograrse o bien reimplantando o suplementado periódicamente aquellas especies bacterianas con efectos positivos (mediante probióticos), o bien incrementando la ingesta de sustratos que hagan crecer dichas especies bacterianas (mediante prebióticos).

\section{Bacterias con efecto negativo sobre la ansiedad}

En los últimos años, se ha documentado que algunos patógenos tradicionales, como Escherichia spp. o Shigella spp. tienen una mayor presencia en personas con trastornos psicológi$\cos (9)$. En general, la mayor parte de las exotoxinas se encuentran en mayor cantidad cuando además de existir una menor diversidad en la MI, ésta se encuentra dominada por bacterias del filo Proteobacteria, especialmente de la familia de las gamma-proteobacterias (44). Precisamente, esta familia es la que habitualmente se ve más favorecida como consecuencia del uso de los antibióticos de amplio espectro. Esto se produce porque aunque las proteobacterias representan en un individuo adulto sano aproximadamente el $15 \%$ de la MI (45), éstas poseen aproximadamente el $35 \%$ de los genes y transposones codificantes de resistencia a los distintos tipos de antibióticos que están incluidos en el microbioma (1). Por el contrario, el filo Bacteriodetes, que debería ser el predominante en la MI de los individuos adultos sanos, sólo acumula el $6 \%$ de los elementos genéticos que codifican mecanismos de resistencia a los antibióticos $(45,46)$.

Entre los grupos bacterianos que más se han caracterizado como agentes disbióticos por causar alteraciones en la mucosa intestinal destacan algunas especies del género Clostridium, en concreto las especies difficile, histolyticum, bolteae y tetani (28). Otro importante grupo es el género Fusobacterium, bacteria patógena que es capaz de producir un efecto inflamatorio crónico y que está muy relacionada con el desarrollo del cáncer de colon (47), especialmente la especie Fusobacterium nucleatum (48).

También existen algunas especies, como Ruminococcus gnavus, que por su capacidad de degradar las mucinas gastrointestinales (47) puede llegar a causar una desprotección del epitelio intestinal, dejándolo así mucho más vulnerable al ataque de bacterias patógenas. Adicionalmente, si bien no se ha demostrado de manera específica su acción negativa sobre el SNC, sí se ha podido demostrar que los pacientes con trastornos de ansiedad presentan mayores concentraciones de dos especies bacterianas concretas: Bacteroides dorei y Coprococcus eutactus, por lo que se ha postulado que deben tener algún efecto inductor de la ansiedad (9). Otros patógenos tradicionales, como Campylobacter jejuni también han demostrado en modelos animales aumentar el comportamiento similar a la ansiedad (17).

\section{Bacterias con efecto positivo sobre la ansiedad}

$\mathrm{Al}$ contrario que lo expuesto en el apartado anterior, y en base a los ya mencionados mecanismos de comunicación entre la MI y en SNC, podemos deducir que tendrán un efecto beneficioso todas aquellas especies que promuevan la sintesis de AGCC, esenciales en el mantenimiento de la barrera intestinal. De hecho, se ha encontrado una importante disminución de la abundancia de cinco géneros productores de AGCC (Faecalibacterium, Eubacterium rectale, Lachnospira, Butyricicoccus y Sutterella) en sujetos con trastorno de ansiedad generalizada (49).

Además de su importante efecto sobre el manteamiento de la permeabilidad 
intestinal, los AGCC también pueden potenciar la actividad de los receptores acoplados a la proteína $\mathrm{G}(50)$, promoviendo la diferenciación de ECCs (51). En el SNC, los AGCC tienen además efecto neuroprotector. El ácido butírico, en forma de butirato de sodio incrementa la expresión del factor neurotrófico derivado del cerebro, mejorando de este modo las actividades relacionadas con la memoria (17). Por ello, la ingesta de probióticos que aumentan la fermentación bacteriana de los alimentos tiene resultados positivos en la salud mental del paciente. Además de los AGCC, existen numerosos neurometabolitos secretados directamente por la MI y producidos por la acción estimulante de la MI sobre las células epiteliales secretoras (51).

\section{Efectos positivos de los probióticos en el tratamiento de la ansiedad}

Las principales características que deben reunir los probióticos incluyen ser seguros, resistentes a los ácidos, a las sales biliares y ser capa- ces de colonizar el tracto digestivo. Los probióticos más utilizados para realizar estudios sobre sus beneficios son las bacterias ácido-lácticas (LAB), que incluyen especies de los géneros Lactobacillus, Enterococcus, Streptococcus y Bifidobacterium (52). El motivo principal por el que las LAB son probióticos, es su capacidad para adherirse a las paredes intestinales, colonizarlas y producir la liberación de ciertas hormonas participantes en la regulación de la ingesta como el CCK y el GLP-1. De entre todas ellas, trabajos recientes han señalado que son las cepas de Lactobacillus spp. las que mejor resisten las condiciones del tracto digestivo, destacando especialmente la especie L. gasseri (52). Por último, las cepas probióticas que demuestran mayor capacidad de adherencia a las paredes intestinales son $L$. casei y $L$. fermentum (53).

Entre los estudios que han sido publicados referentes al uso de probióticos en el manejo y control de la ansiedad, la mayor parte fueron realizados utilizando modelos animales, como los ratones, y muy pocos en personas. Esto se debe al principio de prudencia a la hora de realizar ensayos clínicos, ya que siempre es necesario por motivos éticos demostrar la ausencia de efectos adversos en modelos animales antes realizar ensayos en personas (52). No obstante, los mecanismos fisiológicos en animales y humanos no son iguales, por lo que los resultados no son siempre extrapolables (54).

Entre los estudios específicos para el control de la ansiedad, podemos ver un resumen de sus resultados en la tabla 1.

Uno de los probióticos que ha demostrado efectos ansiolíticos es $L$. rhamnosus, efecto que consigue mediante un mecanismo de inhibición de la activación vagal (55). También se han demostrado efectos reguladores del estrés y la ansiedad mediante modificaciones neuroquímicas en animales de experimentación después de la suplementación con bacterias probióticas del grupo de las bifidobacterias, como el B. longum 1714, B. breve 1205 (56).

Tabla 1 Resultados de los estudios con efectos positivos sobre probióticos para el manejo de la ansiedad

\begin{tabular}{|c|c|c|c|c|}
\hline Autor & Año & Cepa probiótica & Tipo de ensayo & Efecto observado \\
\hline (25) & 2018 & L. rhamnosus JB-1 & $\begin{array}{l}\text { Ensayo en animales } \\
\text { experimentación }\end{array}$ & $\begin{array}{l}\text { Mejora de las crisis de ansiedad y comportamiento anti- } \\
\text { social, además de reducir la secreción de cortisol inducida } \\
\text { por el estrés. }\end{array}$ \\
\hline$(52)$ & 2016 & $\begin{array}{l}\text { L. gasseri CMUL34 y L. } \\
\text { acidophilus CMUL67 }\end{array}$ & $\begin{array}{l}\text { Ensayo experimental } \\
\text { in vitro }\end{array}$ & $\begin{array}{l}\text { L. gasseri CMUL34 y L. acidophilus CMUL67 son capaces } \\
\text { de modular in vitro la expresión de los GLP y de la CCK. }\end{array}$ \\
\hline (54) & 2011 & $\begin{array}{l}\text { L. helveticus R0052 y } B \text {. } \\
\text { longum R0175 }\end{array}$ & $\begin{array}{l}\text { Ensayo en animales } \\
\text { experimentación y en } \\
\text { humanos }\end{array}$ & $\begin{array}{l}\text { Disminución del estrés y la ansiedad en animales de expe- } \\
\text { rimentación y adultos hospitalizados mediante diversas } \\
\text { escalas. }\end{array}$ \\
\hline (55) & 2011 & L. rhamnosus & $\begin{array}{l}\text { Ensayo en animales } \\
\text { experimentación }\end{array}$ & $\begin{array}{l}\text { Efectos ansiolíticos mediante la inhibición del reflejo va- } \\
\text { gal. }\end{array}$ \\
\hline (56) & 2014 & $\begin{array}{l}\text { B. longum } 1714 \text { y B. brevis } \\
1205\end{array}$ & $\begin{array}{l}\text { Ensayo en animales } \\
\text { experimentación }\end{array}$ & $\begin{array}{l}\text { Disminución de la ansiedad en ratones con ansiedad inducida } \\
\text { mediante la inhibición de vías neuronales específicas. }\end{array}$ \\
\hline (57) & 2015 & $\begin{array}{l}\text { Combinado de cepas aislados } \\
\text { de yogur }\end{array}$ & $\begin{array}{l}\text { Ensayo en } 70 \text { personas } \\
\text { adultas sanas }\end{array}$ & $\begin{array}{l}\text { Mejora de los síntomas de ansiedad a través del eje hipo- } \\
\text { talámico-hipófisis-adrenal. }\end{array}$ \\
\hline (58) & 2016 & $\begin{array}{l}\text { Mezcla de L. acidophilus, L. } \\
\text { casei y B. Bifidum }\end{array}$ & $\begin{array}{l}\text { Ensayo en } 70 \text { personas } \\
\text { adultas sanas }\end{array}$ & $\begin{array}{l}\text { Mejora de los sintomas de ansiedad, depresión y estado } \\
\text { de ánimo en general. }\end{array}$ \\
\hline (59) & 2014 & Cl. butyricum & $\begin{array}{l}\text { Ensayo en } 30 \\
\text { pacientes recién } \\
\text { operados de cáncer }\end{array}$ & $\begin{array}{l}\text { Reducciones estadisticamente significativas en los niveles } \\
\text { de ansiedad en el grupo tratado con probióticos versus el } \\
\text { grupo de control. }\end{array}$ \\
\hline (60) & 2014 & $\begin{array}{l}\text { L. gasseri SBT2055 y } B . \\
\text { longum SBT } 2928\end{array}$ & $\begin{array}{l}\text { Ensayo en } 224 \\
\text { personas adultas sanas }\end{array}$ & $\begin{array}{l}\text { Mejora de los sintomas de ansiedad mediada por la } \\
\text { mejora en la actividad de las células "natural-killer". }\end{array}$ \\
\hline (61) & 2015 & $\begin{array}{l}\text { Combinación de probióticos } \\
\text { (B. bifidum W23, B. lactis } \\
\text { W52, L. acidophilus W37, L. } \\
\text { brevis W63, L. casei W56, L. } \\
\text { salivarius W24 y L. lactis W19 } \\
\text { y W58) }\end{array}$ & $\begin{array}{l}\text { Ensayo en } 40 \text { personas } \\
\text { adultas sanas }\end{array}$ & $\begin{array}{l}\text { Mejora de los sintomas de ansiedad, depresión y estado } \\
\text { de ánimo en general. }\end{array}$ \\
\hline
\end{tabular}

CCK: Colecistoquinina; GLP-1: Péptido de glucagon 1 
Con respecto a los ensayos clínicos en humanos, existen algunos en los que la suplementación probiótica mejoró significativamente la sintomatología de ansiedad. A este respecto, podemos mencionar el realizado por Mohammadi y col. (57), en el que demostró que cápsulas que contenían un grupo de agentes bacterianos aislados a partir de yogur mejoraban los sintomas de ansiedad a través del eje hipotalámico-hipófisis-adrenal. En otro trabajo, Messaoudi y col. (54) demostraron mejoras en los niveles de ansiedad y estrés mediante la suplementación de L. helveticus R0052 y B. longum R0175 durante seis semanas. De manera similar, Steenbergen y col. (61) demostraron una importante mejora en los pacientes tratados mediante la administración durante un período de cuatro semanas de una combinación de probióticos, que contenía B. bifidum W23, B. lactis W52, L. acidophilus W37, L. brevis W63, L. casei W56, L. salivarius W24 y L. lactis (W19 y W58).

Posteriormente, Akkasheh y col. (58) demostraron mediante un ensayo clínico en el que participaron un total de 40 pacientes deprimidos que la administración durante ocho semanas de una mezcla probiótica que contenía L. acidophilus, L. casei, y $B$. bifidum mejoró los síntomas relacionados con la depresión con respecto a los sujetos que actuaron como controles.

Por su parte, Yang y col. (59) estudiaron el efecto de dos semanas de suplementación con $\mathrm{Cl}$. butyricum en pacientes con ansiedad pre-quirúrgica, y también encontraron reducciones estadísticamente significativas en el nivel de ansiedad con respecto a los sujetos que no recibieron suplementación probiótica. Cabe destacar que los pacientes que recibieron placebo, no sólo mantuvieron intactos sus niveles de ansiedad, sino que los incrementaron de manera significativa.

El uso de un probiótico a base de L. rhamnosus $\mathrm{JB}-1$ ha demostrado la mejora de las crisis de ansiedad y comportamiento antisocial en ratones (25). Este probiótico demostró capacidad de reducir la secreción de cortisol inducida por el estrés. Por otra parte, se ha visto que la administración de leche fermentada que contenía diferentes probióticos, alteró la actividad cerebral de las regiones asociadas a la emoción en voluntarias sanas, su- giriendo una posible acción potencial psicobiótica (25).

Un estudio clínico en humanos, realizado por Messaoudi y col. (54) en un grupo de 55 personas, comparó la administración a un grupo de personas seleccionada de manera aleatoria un combinado probiótico formado por L. helveticus R0052 y B. longum R0175 y a otros placebos por un periodo de 30 días. Los resultaros fueron que aquellos que tomaron el combinado demostraron mejorar los síntomas de ansiedad. Por el contrario, también existen trabajos que no encontraron beneficios asociados a la ingesta de probióticos en la ansiedad (11). En su mayor parte, dichos trabajos fueron realizados a principios de la década de 2000, cuando la tecnología tanto de elaboración de probióticos como de estudio de la MI estaban lejos de las condiciones actuales (11). Ninguno de los ensayos realizados encontró efectos negativos como consecuencia de la ingesta de probióticos, por lo que puede deducirse de que si bien no puede asegurarse al cien por cien un efecto beneficioso, la administración de dichos probióticos es segura (11).

\section{Efectos positivos de los prebióticos en el tratamiento de la ansiedad}

Los agentes prebióticos más habitualmente utilizados son los fructanos, como la inulina y los fructooligosacáridos (FOS), los galactooligosacáridos (GOS) y las ciclodextrinas (62). En todos los casos pueden ser o bien componentes naturales incluidos en algunos alimentos vegetales o bien sustancias producidas industrialmente (62).

Un resumen de los trabajos realizados para evaluar la eficacia de los prebióticos en los trastornos de ansiedad podemos observarlo en la tabla 2. En modelos animales, algunos agentes prebióticos han demostrado poseer efectos positivos sobre algunas patologías neurológicas. Por ejemplo, se ha podido determinar que la suplementación con GOS no digeribles en ratones tratados con LPS consigue mejoras en el comportamiento típico de ansiedad (63). Otro trabajo que obtuvo resultados interesantes fue el publicado por Burokas y col. (64), en el que comprobaron cuales eran los efectos de la administración general de prebióticos en ratones. Se realizaron tres grupos distintos para el estudio: ratones a los que se les administró una combinación de FOS+GOS, otro grupo al que se le administró FOS y otro GOS. En el grupo de FOS+GOS observaron que no se producía ninguna variación en la ganancia de peso ni en los niveles de glucosa en plasma pero sí en la ganancia de peso del ciego después de un periodo de diez semanas administrando probióticos. Observaron que la MI predominante en el ciego de los ratones era Lanchnospiraceae. También se observaron altos niveles de Verrucomicrobiaceae, Bacteroides Paracateroides, y Oscillibacter solo en el grupo de FOS. Los resultados obtenidos demostraron que la administración prebiótica producía un incremento del número de bacterias distintas, sin afectar a las cantidades presentes de Lactobacillus y Bifidobacterium.

Para medir el grado de ansiedad en ratones se realizó una prueba de campo abierto. Esta prueba consiste en evaluar la reacción de los sujetos a un acontecimiento estresante midiendo la cantidad de estímulos estresantes que los ratones emiten cuando se encuentran en una superficie expuesta e iluminada (64). Mediante esta prueba pudo observarse que la administración de FOS+GOS aumentaba la duración del tiempo que pasaban en el centro y su número de entradas a él, pero no hubo ninguna diferencia significativa en el periodo de latencia en la zona central (65). Por otra parte, la prueba de laberinto elevado en cruz, es una de las pruebas preclínicas de respuesta más utilizadas en el estudio de la ansiedad y consiste en ver cómo animales de experimentación pasan por un circuito en $\mathrm{X}$ en el cual dos de las salidas del laberinto tienen los finales abiertos y otras dos las salidas cerradas, en las que se mide el tiempo que los animales pasan en los pasillos expuestos y en los cerrados (64). Mediante este método, observó que al administrar la combinación de FOS+GOS los ratones mostraban una tendencia al enterramiento mucho menor que en el grupo control. También se observó la reducción de los niveles de L-triptófano en plasma y, a su vez, un aumento de los niveles de serotonina en el córtex prefrontal (65). 
Tabla 2 Resultados de los estudios con efectos positivos sobre prebióticos para el manejo de la ansiedad

\begin{tabular}{|c|c|c|c|c|}
\hline Autor & Año & Prebiótico & Tipo de ensayo & Efecto observado \\
\hline (43) & 2014 & GOS & $\begin{array}{l}\text { Ensayo en animales } \\
\text { experimentación }\end{array}$ & $\begin{array}{l}\text { Incrementa niveles intestinales de Bifidobacterium spp. } \\
\text { y Lactobacillus spp., otras } L A B \text {, además de otras como } \\
\text { Actinobaterium spp., y Bacteroides spp. Redujo la secreción de } \\
\text { citoquinas proinflamatorias y cortisol e incrementó la liberación } \\
\text { de citoquinas antiinflamatorias. }\end{array}$ \\
\hline (63) & 2012 & GOS & $\begin{array}{l}\text { Ensayo en animales } \\
\text { experimentación }\end{array}$ & Mejora en el comportamiento típico de ansiedad \\
\hline (65) & 2017 & FOS + GOS & $\begin{array}{l}\text { Ensayo en animales } \\
\text { experimentación }\end{array}$ & $\begin{array}{l}\text { El combinado FOS+GOS favorece el incremento de bacterias } \\
\text { con efecto positivo pero no afecta a Lactobacillus spp. y } \\
\text { Bifidobacterium spp. Los ratones tratados experimentaron } \\
\text { una disminución en los niveles de corticosterona y citoquinas } \\
\text { proinflamatorias. }\end{array}$ \\
\hline (66) & 2018 & Mezcla de GOS & $\begin{array}{l}\text { Ensayo en animales } \\
\text { experimentación }\end{array}$ & $\begin{array}{l}\text { La administración de GOS favorece la proliferación colónica } \\
\text { de Bifidobacterias, provocando una mejoría en el estado } \\
\text { neuroinflamatorio y cognitivo. }\end{array}$ \\
\hline (74) & 2016 & Pectina de manzana & $\begin{array}{l}\text { Ensayo en animales } \\
\text { experimentación }\end{array}$ & $\begin{array}{l}\text { Incrementa la diversidad de la } \mathrm{Ml} \text {, al tiempo que redujo la } \\
\text { endotoxemia y numerosos marcadores de inflamación, incluidos } \\
\text { a nivel neuronal. }\end{array}$ \\
\hline (75) & 2012 & $\begin{array}{l}\text { Dieta con alto contenido } \\
\text { en fibras prebióticas }\end{array}$ & $\begin{array}{l}\text { Ensayo en animales } \\
\text { experimentación } \\
\text { y en humanos }\end{array}$ & $\begin{array}{l}\text { Una dieta con elevado contenido en fibras prebióticas provocó } \\
\text { una mayor secreción de hormonas intestinales inductoras de la } \\
\text { saciedad y de AGCC }\end{array}$ \\
\hline
\end{tabular}

FOS: Fructooligosacáridos; GOS: Galactooligosacáridos; LAB: bactérias ácido-lácticas; MI: Microbiota intestinal.

Otro trabajo interesante acerca del papel de los prebióticos en la regulación de la ansiedad fue el realizado por Yang y col. (66), mediante el cual se demostró que la administración de $\beta$-galactosacáridos ( $\beta$-GOS) inducía un cambio significativo en la diversidad de la MI y la proliferación de Bifidobacterium y otros microbios potencialmente antiinflamatorios. Resultados más modestos se han conseguido al suplementar a animales de experimentación con pectina, aumentando la diversidad en la MI, al tiempo que redujo la endotoxemia y numerosos marcadores de inflamación (41).

Existen pocos trabajos realizados en humanos que hayan estudiado el efecto de los prebióticos de manera aislada sobre la influencia que ésta pueda tener sobre la ansiedad. Entre estos destaca el realizado por Geurts y col. (43). En uno de estos estudios los GOS demostraron incrementar los niveles intestinales de Bifidobacterium spp. y Lactobacillus spp., y otras LAB. Dichas bacterias están consideradas como inductoras de un efecto inmunomodulador, reduciendo la secreción de citoquinas proinflamatorias y aumentando la liberación de citoquinas antiinflamatorias. La administración durante tres semanas de $\beta$-GOS ha supuesto una bajada de niveles de cortisol en pacientes con ansiedad, lo que permite valorar su administración como una posible opción terapéutica.

\section{Ventajas de la administración de prebióticos $\mathrm{y}$ probióticos a través de presentaciones farmacéuticas}

Dado que el principal agente modulador de la MI es la dieta, por ser capaz de variar su composición de una manera rápida (21), existe la tendencia a creer que la mejor manera de modificar la MI para corregir los estados de disbiosis debe hacerse modificando la dieta o incluyendo tanto los prebióticos como los probióticos a través de los alimentos. No obstante, los consumidores suelen mostrarse reticentes a cambiar de un modo importante sus hábitos alimentarios cuando se le pide además que dichos cambios sean sostenidos por largos períodos de tiempo (67). Por ello, en lugar de tratar de modificar totalmente la MI, podemos conseguir beneficios estimulando el crecimiento de especies bacterianas beneficiosas mediante prebióticos o inocular especies probióticas. Los alimentos más habitualmente empleados para vehicular probióticos a los consumidores son los productos lácteos fermentados y las bebidas de frutas (68). No obstante, estos productos tienen el inconveniente de que requieren una refrigeración constante (69) y al ser matrices con elevada actividad de agua, la supervivencia de los probióticos en ellos es limitada (68).

Para ejercer un efecto beneficioso sobre el organismo, tanto los probióticos como los prebióticos deben ser administrados en dosis adecuadas, y es esencial mantener su viabilidad hasta el momento de su consumo (68). Por ello, una alternativa para su administración son las presentaciones farmacéuticas, en las cuales ambos agentes se mantienen generalmente de manera deshidratada. De este modo, los microorganismos probióticos tienen una conservación más adecuada y duradera que si se encuentran en matrices con más actividad del agua como los alimentos (70). Además, cada prebiótico y probiótico deben ser ingeridos en dosis específicas para poder ejercer su efecto beneficioso de manera efectiva, lo cual es difícil de controlar a través 
de alimentos, ya que cada consumidor los ingiere en una cantidad diferente (67). En el caso de los probióticos, su efectividad también depende de su supervivencia durante el tránsito por la parte anterior del sistema digestivo humano (69). El grado de acidez del estómago humano constituye un ambiente hostil para la mayor parte de los microorganismos probióticos. Por ello, si se ingieren mediante alimentos, el pH del estómago dependerá del volumen de ingesta alimentaria y buena parte de los probióticos pueden ser destruidos (69). Por el contrario, si se ingieren a través de presentaciones farmacéuticas, el consejo farmacéutico le orientará de manera eficaz para una adecuada toma y los probióticos mantendrán su actividad.

También se asegura una mejor estabilidad de los microrganismos independientemente de su naturaleza. Algunos agentes probióticos que han sido relacionados con el manejo de la ansiedad requieren una atmósfera especial, como son Bifidobacterium o los Clostridium, que son anaerobios estrictos (70). Por este motivo, su administración a través de los alimentos presenta mayores dificultades que a través de presentaciones farmacéuticas.

Como ventaja añadida, el uso de presentaciones farmacéuticas para este fin permite al usuario acceder al consejo farmacéutico, lo que sin duda alguna le aporta un valor adicional a esta vía de administración (71). Si nos ceñimos a su empleo en el manejo específico de la ansiedad, el uso de antibióticos puede potenciar de manera importante los síntomas de ansiedad, llegando incluso a provocar crisis agudas (72). Por ello, una primera medida que podríamos implementar desde el punto de vista de la farmacia comunitaria para reducir la predisposición a los trastornos de ansiedad es evitar la ingesta de antibióticos sin acompañarlos de probióticos que amortigüen su efecto.

Por otra parte, el hecho de que los tratamientos farmacológicos empleados para tratar la ansiedad deban ser retirados de una manera gradual y pautada cuando los sintomas remi$\tan (9)$, también hace que sea útil que desde la farmacia comunitaria recomendemos a nuestros pacientes una suplementación probiótica cuando inicie el proceso de retirada del tratamiento farmacológico prescrito, a fin de atenuar los inconvenientes que ocurren cuando se reduce la dosis.
El consejo farmacéutico ha demostrado en otros ámbitos mejorar la adherencia a los tratamientos por parte del paciente, su toma correcta y reduce su abandono precoz (71). Si el probiótico es recomendado por un médico, estudios previos han demostrado que frecuentemente los pacientes no comprenden en su totalidad lo que el médico les dice (73). Si, por el contrario, la recomendación llega por otra diferente como son los medios de comunicación, la posibilidad de que la información que le ha llegado al paciente sea incorrecta es mucho mayor (71) y el consejo farmacéutico será aún más valioso.

\section{Conclusiones}

Según la información científica expuesta, queda claro que la MI puede influir en la actividad del SNC a través de múltiples mecanismos. En concreto, la MI también muestra efectos específicos sobre la ansiedad. La modificación de la MI puede abordarse a través de probióticos 0 prebióticos administrados en presentaciones farmacéuticas, las cuales presentan ventajas con respecto a la suplementación de alimentos como un mejor control de las dosis, un menor rechazo por parte del paciente (no implican cambios de hábitos alimentarios) y un menor abandono precoz de los tratamientos. Adicionalmente, en algunas especies de probióticos como los Bifidobacterium, su administración es más sencilla, ya que al ser anaerobios estrictos su supervivencia en alimentos es más compleja. Si bien no todos los ensayos realizados han mostrado efectos positivos, en ninguno de ellos se ha encontrado ningún efecto negativo, por lo que puede concluirse que su administración es segura. Los más habituales con efectos sobre la ansiedad pertenecen a los géneros Lactobacillus y Bifidobacterium, aunque también algunas especies de Clostridium han demostrado efectos positivos. Existen especies con efectos negativos, como Escherichia coli, Fusobacterium nucleatum o Ruminococcus gnavus que deben prevenirse y/o limitarse su acción patógena.

Algunos agentes prebióticos también han mostrado efectos positivos sobre la ansiedad, principalmente en animales de experimentación. Su administración, conjuntamente con pro- bióticos o de manera individual, también es una opción terapéutica útil.

Si bien la suplementación de alimentos es la vía más habitual para la administración de probióticos y prebióticos, la administración a través de presentaciones farmacéuticas presenta muchas ventajas sobre los alimentos referente a la estabilidad de los mismos, o la posibilidad de tomarlos sin alterar significativamente sus hábitos dietéticos. Del mismo modo, la posibilidad de acceso al consejo farmacéutico es un valor añadido para los consumidores, que ayuda a una correcta administración y adherencia del tratamiento.

\section{Referencias bibliográficas}

1. Roca-Saavedra P, Mendez-Vilabrille V, Miranda JM, Nebot C, Cardelle-Cobas A, Franco CM, Cepeda C. Food additives, contaminants and other minor components: Effects on human gut microbiota-a review. Journal of Physiology and Biochemistry. 2018; 74(1), 69-83. doi:10.1007/s13105017-0564-2

2. De Filippo C, Cavalieri D, Di Paola M, Ramazzotti M, Poullet JB, Massart S, Collini, S, Pieraccini G, Lionetti P. Impact of diet in shaping gut microbiota revealed by a comparative study in children from Europe and rural Africa. Proceedings of the National Academy of Sciences of the United States of America. 2010; 107, 14691-14696. doi:10.1073/pnas. 1005963107

3. Cryan JF, Dinan TG. Mind-altering microorganisms: the impact of the gut microbiota on brain and behaviour. Nature Reviews in Neuroscience. 2012; 13(10), 701-712. doi:10.1038/ nrn3346

4. Conlon MA, Bird AR. The impact of lifestyle on gut microbiota and human health. Nutrients. 2015; 7(1), 1744. doi:10.3390/nu7010017

5. García-Mantrana I, Selma-Royo M, Alcantara C, Collado MC. Shift son gut microbiota associated to Mediterranean diet adherence and specific dietary intakes on general adult population. Frontiers in Microbiology. 2018; 9, 890. doi:10.3389/fmicb.2018.00890

6. Cresci GA, Bawden E. Gut microbiome: What we do and don't know. Nutrition in Clinical Practice, 2016; 30, 734746. doi:10.1177/0884533615609899

7. Schippa S, Conte MP. Dysbiotic events in gut microbiota: Impacts on human health. Nutrients. 2014; 6, 5786-5805. doi:10.3390/nu6125786

8. Macedo D, Filho A, Soares de Sousa CN, Quevedo J, Barichello T, Junior 
HVN, Freitas de Lucena, D. Antidepressants, antimicrobials or both? Gut microbiota dysbiosis in depression and possible implications of the antimicrobial efects of antidepressant drugs for antidepressant efectiveness. Journal of Afective Disorders. 2017; 208, 22-32. doi:10.1016/j.jad.2016.09.012

9. Jiang HY, Zhang X, Yu ZH, Zhang Z, Deng M, Zhao JH, Ruan B. Altered gut microbiota profile in patients with generalized anxiety disorder. Journal of Psychiatric Research. 2018; 104, 130-136. doi:10.1016/j.jpsychires.2018.07.007

10. Akyol A, Ayaz A, Inan-Eroglu E, Cetin C, Samur G. Impact of three different plate colours on short-term satiety and energy intake: a randomized controlled trial. Nutrition Journal. 2018; 17, 46. doi:10.1186/s12937018-0350-1

11. Pirbaglou M, Katz J, de Souza RJ, Stearns JC, Motamed M, Rivo P. Probiotic supplementation can positively affect anxiety and depressive symptoms: a systematic review of randomized controlled trials. Nutrition Research. 2016; 36, 889-898. doi:10.1016/j.nutres.2016.06.009

12. Heric GA, Little TJ, Kohn M, Madden S, Clarke S, Horowitz M, Feinle-Bisset C. Appetite perceptions, gastrointestinal symptoms, ghrelin, peptide YY and state anxiety are disturbed in adolescent females with anorexia nervosa and only partially restored with short-term. Nutrients. 2018; 11, 59. doi:10.3390/nu11010059

13. Foster JA, McVey Neufeld KA. Gut-brain axis: how the microbiome influences anxiety and depression. Trends in Neuroscience. 2013; 36(5), 305-312. doi:10.1016/j. tins.2013.01.005

14. Wang Y, Kasper LH. The role of microbiome in central nervous system disorders. Brain, Behavior and Immunity. 2014; 38, 1-12. doi:10.1016/j. bbi.2013.12.015

15. Zvolensky M, Jardin C, Farris SG, Kauffman B, Bakhsaie J, Garey L, Manning K, Rogers AH, Mayorga NA. Gut interpretations: how difficulties in emotion regulation may help explain the relation of visceral sensitivity with depression and anxiety among young adults with gastrointestinal symptoms. Psychology, Health and Medicine. 2018; 23(7), 840-845. doi:1 $0.1080 / 13548506.2018 .1455984$

16. Sherwin E, Dinan TG, Cryan JF. Recent developments in understanding the role of the gut microbiota in brain health and disease. Annals of the New York Academy of Sciences. 2018; 1420, 5-25. doi:10.1111/nyas. 13416

17. Kim JS, de la Serre CB. Diet, gut microbiota composition and feeding behavior. Physiology and Behavior. 2018; 192, 177-181. doi:10.1016/j. physbeh.2018.03.026

18. Lew LC, Hor YY, Asmaa N, Yusoff A, Choi SB, Tusoff MSB, Roslan NS, Ahmad A, Mohammad JAM, Abdullah MFIL, Zakaria N, Wahid N, Sun Z, Kwok LL, Zhang H, Liong MT. Probiotic Lactobacillus plantarum P8 alleviated stress and anxiety while enhancing memory and cognition in stressed adults: A randomized, double-blind, placebo-controlled study. Clinical Nutrition, 2018. doi:10.1016/j. clnu.2018.09.010

19. Westfall S, Lomis N, Kahouli I, Yuan Dia S, Patrap Singh S, Prakash S. Microbiome, probiotics and neurodegenerative diseases: deciphering the gut brain axis. Cellular and Molecular Life Sciences. 2017; 74, 3769-3787. doi:10.1007/s00018-017-2550-9

20. Sampson TR, Debelius JW, Thron T, Janssen S, Shastri GG, Illan ZE, Challis C, Schretter CE, Rocha S, Gradinaru V, Chesselet MF, Keshavarzian A, Shannon KM, Krajmalnik-Brown R, Wittung-Stafshede P, Knight R, Mazmanina SK. Gut microbiota regulate motor deficits and neuroinflammation in a model of Parkinson's disease. Cell. 2016; 167(1469-1480), e12. doi:10.1016/j. cell.2016.11.018

21. De La Serre CB, de Lartigue G, Raybould HE. Chronic exposure to low dose bacterial lipopolysaccharide inhibits leptin signaling in vagal aferent neurons, Physiology and Behaviour. 2015; 139, 188-194. doi:10.1016/j. physbeh.2014.10.032

22. Bruce-Keller AJ, Salbaum JM, Luo M, Blanchard E, Taylor CM, Welsh DA, Berthoud HR. Obese-type gut microbiota induce neurobehavioral changes in the absence of obesity. Biological Psychiatry. 2015; 77, 607-615. doi:10.1016/j.biopsych.2014.07.012

23. Cani PD, Everard A. Talking microbes: When gut bacteria interact with diet and host organs. Molecular Nutrition and Food Research. 2016; 60, 58-66. doi:10.1002/mnfr.201500406

24. Sen T, Cawthon CR, Ihde BT, Hajnal A, di Lorenzo P, de la Serra CB, Czaja $\mathrm{K}$. Diet-driven microbiota dysbiosis is associated with vagal remodeling and obesity. Physiology and Behavior. 2017; 173, 305-317. doi:10.1016/j. physbeh.2017.02.027

25. Dinan TG, Cryan JF, Stanton C. Gut microbes and brain development have black box connectivity. Biological Psychiatry. 2018; 83(2), 97-99. doi:10.1016/j.biopsych.2017.11.005

26. Dryman MT, Heimberg RG. Emotion regulation in social anxiety and depression: a systematic review of expressive suppression and cognitive reappraisal. Clinical Psychology Reviews. 2018; 65, 17-42. doi:10.1016/j. cpr.2018.07.004

27. Noble EE, Hsu TM, Kanoski SE. Gut to brain dysbiosis: mechanisms linking western diet consumption, the microbiome, and cognitive impairment. Frontiers in Behavioral Neuroscience. 2017; 11:9. doi:10.3389/ fnbeh.2017.00009

28. Hamilton MK, Boudry G, Lemay DG, Raybould HE. Changes in intestinal barrier function and gut microbiota in high-fat diet-fed rats are dynamic and region dependent, American Journal of Physiology, Gastrointestinal and Liver Physiology. 2015; 308, G840-G851. doi:10.1152/ajpgi.00029.2015

29. O'Loughlin E, Pakan JMP, Yilmazer-Hanke D, McDermott KW. Acute in utero exposure to lipopolysaccharide induces inflammation in the pre- and postnatal brain and alters the glial cytoarchitecture in the developing amygdala. Journal of Neuroinflammation. 2017; 14, 212. doi:10.1186/s12974-017-0981-8

30. Mayer EA, Knight R, Mazmanian SK, Cryan JF, Tillisch K. Gut microbes and the brain: paradigm shift in neuroscience. Journal of Neuroscience. 2014 34(46), 15490-15496. doi:10.1523/ JNEUROSCI.3299-14.2014

31. Rogers GB, Keating DJ, Young RL, Wong ML, Licinio J, Wesselingh S. From gut dysbiosis to altered brain function and mental illness: mechanisms and pathways. Molecular Psychiatry. 2016; 21, 738-748. doi:10.1038/mp.2016.50

32. Reutner L, Genschow 0, Wänke M. The adaptative eater: perceived healthiness moderates the effect of the color red on consumption. Food Quality and Preferences. 2015; 44, 172-178. doi:10.1016/j.foodqual.2015.04.016

33. Benoit C, Ould-Hamouda H, Crepin D, Gertler A, Amar L, Taouis M. Early leptin blockade predisposes fatfed rats to overweight and modifies hypothalamic microRNA. Journal of Endocrinology, 2013; 12, 35-47. doi:10.1530/JOE-12-0561

34. Crépin D, Benomar Y, Riffault L, Amine H, Gertler A, Taouis M. The over-expression of miR-200a in the hypothalamus of ob/ob mice is linked to leptin and insulin signaling impairment. Molecular and Cellular Endocrinology. 2014; 384(1), 1-11 doi:10.1016/j.mce.2013.12.016

35. Sangiao-Alvarellos S, Pena-Bello L, Manfredi-Lozano M, Tena-Sempere M, Cordido F. Perturbation of hypothalamic microRNA expression patterns in male rats after metabolic distress: impact of obesity and conditions of negative energy balance. 
Endocrinology. 2014; 155(5), 18381850. doi:10.1210/en.2013-1770

36. Derghal A, Djelloul M, Trouslard J, Mounien L. The role of microrna in the modulation of the melanocortinergic system. Frontiers in Neuroscience. 2017; 11, 181. doi:10.3389/ fnins.2017.00181

37. Price NL, Singh AK, Rotllan N, Goedeke L, Wing A, Canfrán-Duque A, Suárez Y. Genetic ablation of miR33 increases food intake, enhances adipose tissue expansion, and promotes obesity and insulin resistance. Cell Reports. 2018; 22(8), 2133-2145. doi:10.1016/j.celrep.2018.01.074

38. Stevens BR, Goel R, Seungbum K, Richards EM, Holbert RC, Pepine CJ, Raizada MK. Increased human intestinal barrier permeability plasma biomarkers zonulin and FABP2 correlated with plasma LPS and altered gut microbiome in anxiety or depression. Gut. 2018; 8, 1555-1557. doi:10.1136/ gutjnl-2017-314759

39. Xiang Ng Q, Peters C, Xian Ho CY, Lim DY, Yeo WS. A meta-analysis of the use of probiotics to alleviate depressive symptoms. Journal of Affective Disorders. 2018; 228, 13-19. doi:10.1016/j.jad.2017.11.063

40. Sherwin E, Dinan TG, Cryan JF. Recent developments in understanding the role of the gut microbiota in brain health and disease. Annals of the New York Academy of Sciences. 2018; 1420, 5-25. doi:10.1111/nyas.13416

41. Jiang H, Ling Z, Zhang $Y$, Mao H, Ma Z, Yin Y, Wang W, Tang W, Tan Z, Shi J, Li, L, Ruan B. Altered fecal microbiota composition in patients with major depressive disorder. Brain, Behavior and Immunity. 2015; 48, 186194. doi:10.1016/j.bbi.2015.03.016

42. Evans SJ, Bassis CM, Hein R, Assari S, Flowers SA, Kelly MB, Young VB, Ellingrod VE, McInnis MG. The gut microbiome composition associates with bipolar disorder and illness severity. Journal of Psychiatric Research. 2017; 87, 23-29. doi:10.1016/j.jpsychires.2016.12.007

43. Geurts L, Neyrinck AM, Delzenne NM, Knauf C, Cani PD. Gut microbiota controls adipose tissue expansion, gut barrier and glucose metabolism: novel insights into molecular targets and interventions using prebiotics. Wageningen Academic, 2014; 3-17. doi:10.3920/BM2012.0065

44. Kriss M, Hazleto, KZ, Nusbacher NM, Martin CG, Lozupone CA. Low diversity gut microbiota dysbiosis: drivers, functional implications and recovery. Current Opinion in Microbiology, 2018; 44, 34-40. doi:10.1016/j. mib.2018.07.003

45. Hu Y, Yang X, Qin J, Lu N, Cheng G, Wu N, Pan Y, Li J, Wang X, Meng Z,
Zhao F, Liu D, Ma J, Qin N, Xiang C, Xiao Y, Li L, Yan H, Wang J, Yang R, Gao GF, Wang J, Zhu B. Metagenome-wide analysis of antibiotic resistance genes in a large cohort of human gut microbiota. Nature Communications. 2013; 4, 2151. doi:10.1038/ncomms3151

46. Russell SL, Gold MJ, Hartmann M, Willing BP, Thorson L, Wlodarska M, Gill N, Blanchet MR, Mohn, WW, McNagny KM, Finlay BB. Early life antibiotic-driven changes in microbiota enhance susceptibility to allergic asthma. EMBO Reports. 2012; 13, 440447. doi:10.1038/embor.2012.32

47. Dethlefsen L, Eckburg PB, Bik EM, Relman DA. Assembly of the human intestinal microbiota. Trends in Ecology and Evolution. 2006; 21(9), 517523. doi:10.1016/j.tree.2006.06.013

48. Zou S, Fang L, Lee MH. Dysbiosis of gut microbiota in promoting the development of colorectal cancer. Gastroenterology Report. 2018; 6, 1-12. doi:10.1093/gastro/gox031

49. Stilling RM, van de Wouw M, Clarke G, Stanton C, Dinan TG, Cryan JF. The neuropharmacology of butyrate: the bread and butter of the microbiota-gut-brain axis? Neurochemistry International. 2016; 99, 110-132. doi:10.1016/j.neuint.2016.06.011

50. Chang PV, Hao L, Offermanns S, Medzhitov R. The microbial metabolite butyrate regulates intestinal macrophage function via histone deacetylase inhibition. Proceedings of the National Academy of Sciences of the United States of America. 2014; 111, 2247-2252. doi:10.1073/ pnas. 1322269111

51. Knudsen KEB, Laerke HN, Hedemann MS, Nielsen TS, Ingerslev AK, Nielsen DSG, Theil PK, Purup S, Hald S, Schioldan AG, Marco ML, Gregersen S, Hermansen K. Impact of diet-modulated butyrate production on intestinal barrier function and inflammation. Nutrients. 2018; 10, 1499. doi:10.3390/nu10101499

52. Belguesmia Y, Domenger D, Caron J, Dhulster P, Ravallec R, Drider D, Cudennec B. Novel probiotic evidence of lactobacilli on immunomodulation and regulation of satiety hormones release in intestinal cells. Journal of Functional Foods. 2016; 24, 276-286. doi:10.1016/j.jff.2016.04.014

53. Holder MK, Chassaing B. Impact of food additives on the gut-brain axis. Physiology and Behavior. 2018; 192, 173-176. doi:10.1016/j.physbeh.2018.02.025

54. Messaoudi S, Madi A, Prevost H, Feuilloley M, Mania M, Dousset X, Connil N. In vitro evaluation of the probiotic potential of Lactobacillus salivarius SMXD51. Anaerobe. 2012;
18, 584-589. doi:10.1016/j.anaerobe.2012.10.004

55. Bravo JA, Forsythe P, Chew MV, Escaravage E, Savignac HM, Dinan TG, Bienenstock J, Cryan JF. Ingestion of Lactobacillus strain regulates emotional behavior and central GABA receptor expression in a mouse via the vagus nerve, Proceedings of the National Academy of Sciences of the United States of America. 2011; 108, 1605016055. doi:10.1073/pnas. 1102999108

56. Savignac HM, Kiely B, Dina, TG, Cryan FJ. Bifidobacteria exert strain-specific effects on stress-related behavior and physiology in BALC/C mice. Neurogastroenterology and Motility. 2014; 26, 11. doi:10.1111/ nmo.12427

57. Mohammadi A, Jazayeri S, Khosravi-Darani K, Solati Z, Mahammadpour N. The effects pf probiotics on mental health and hypothalamic pituitary-adrenal axis: A randomized, double-blind, placebo-controlled trial in petrochemical workers. Nutritional Neuroscience. 2015; 19, 9. doi:10.117 9/1476830515Y.0000000023

58. Akkasheh G, Kashani-Poor Z, Tajadadi-Ebrahimi M, Jafari P, Akbari $\mathrm{H}$, Taghizadeh M, Memarzadeh MR, Asemi Z, Esmaillzadeh A. Clinical and metabolic response to probiotic administration in patients with major depressive disorder: a randomized, double-blind, placebo-controlled trial. Nutrition. 2016; 32, 315-320. doi:10.1016/j.nut.2015.09.003

59. Yang $\mathrm{H}$, Zhao X, Tang S, Huang $\mathrm{H}$, Ning Z, Fu X, Zhang C. Probiotics reduce psychological stress in patients before laryngeal cancer surgery. Asia-Pacific Journal of Clinical Oncology. 2014; 12, e92-e96. doi:10.1111/ ajco. 12120

60. Nishihira J, Kagami-Katsuyama $\mathrm{H}$, Tanaka A, Nishimura M, Kobayashi T, Kawasaki Y. Elevation of natural killer cell activity and alleviation of mental stress by the consumption of yogurt containing Lactobacillus gasseri SBT2055 and Bifidobacterium longum SBT2928 in a double-blind, placebo controlled clinical trial. Journal of Functional Foods. 2014; 11, 261268. doi:10.1016/j.jff.2014.09.002

61. Steenbergen L, Sellaro R, van Hemert $\mathrm{S}$, Bosch JA, Colzato LS. A randomized controlled trial to test the effect of multispecies probiotics on cognitive reactivity to sad mood. Brain, Behavior and Immunity. 2015; 48, 258264. doi:10.1016/j.bbi.2015.04.003

62. Teresa G, Delgado C, Maria W, Tamashiro C. Role of prebiotics in regulation of microbiota and prevention of obesity. Food Research International. 2018; 113, 183-188. doi:10.1016/j. foodres.2018.07.013 
63. Gopalakrishnan A, Clinthorne JF, Rondini EA, McCaskey SJ, Gurzell EA, Langhohr IM, Gardner EM, Fenton JI. Supplementation with galacto-oligosaccharides increases the percentage of NK cells and reduces colitis severity in Smad3-deficient mice. Journal of Nutrition. 2012; 142(7), 13361340. doi:10.3945/jn.111.154732

64. Polanco, L.A., Vargas-Irwin, C., Góngora, M.E. (2011). Modelos animales: Una revisión desde tres pruebas utilizadas en ansiedad. Suma Psicológica. 18(2), 141-148.

65. Burokas A, Arboleya S, Moloney RD, Peterson VL, Murphy K, Clarke G, Stanton C, Dinan TG, Cryan JF. Targeting the microbiota-gut-brain axis: Prebiotics have anxiolytic and antidepressant-like effects and reverse the impact of chronic stress in mice. Biological Psychiatry. 2017; 82(7), 472-487. doi:10.1016/j. biopsych.2016.12.031

66. Yang XD, Wang LK, Wu HY, Jiao L. Effects of prebiotic galacto-oligosaccharide on postoperative cognitive dysfunction and neuroinflammation through targeting of the gut-brain axis. BMC Anesthesiology. 2018; 18(1), 177. doi:10.1186/s12871-0180642-1
67. Miranda JM, Antón X, Redondo-Valbuena C, Roca-Saavedra P, Rodriguez JÁ, Lamas A, Franco CM, Cepeda A. Egg and egg-derived foods: Effects on human health and use as functional foods. Nutrients. 2015; 7(1), 706-729. doi:10.3390/nu7010706

68. Marcial-Coba MS, Knochel S, Nielsem DS. Low-moisture food matrices as probiotic carriers. FEMS Microbiology Letters. 2019; 366, fnz006. doi:10.1093/femsle/fnz006

69. Sanders ME, Marco ML. Food formats for effective delivery of probiotics. Annual Review of Food Science and Technology. 2010; 1, 65-85. doi:10.1146/annurev.food.080708. 100743

70. El Enshasy H, Malik K, Malek RA, Othman NZ, Elsayed EA, Wadaan M. Anaerobic probiotics: The key microbes for human health. Anaerobes in Biotechnology. 2016; 156, 397-431. doi:10.1007/10_2015_5008

71. Leguelinel-Blache G, Dubois F, Bouvet S, Roux-Marson C, Arnaud F, Castelli C, Ray V, Kinowski JM, Sotto A. Improving patient's primary medication adherence: The value of pharmaceutical counseling. Medicine. 2015; 94(41), e1805. doi:10.1097/ MD.0000000000001805
72. Lurie I, Yang YX, Haynes K, Mantani R, Boursi B. Antibiotic exposure and the risk for depression, anxiety, or psychosis: A nested case-control study. Journal of Clinical Psychiatry. 2015; 76(11), 1522-1528. doi:10.4088/ JCP.15m09961

73. Schillinger D, Piette J, Grumbach K, Wang F, Wilson C, Daher C, LeongGrotz K, Castro C, Bindman AB. Closing the loop: physician communication with diabetic patients who have low health literacy, Archives of Internal Medicine. 2003; 163, 83-90. doi:10.1001/archinte.163.1.83

74. Jiang T, Gao X, Wu C, Tian F, Lei Q, Bi J, Wang HY, Chen S, Wang X. Apple-derived pectin modulates gut microbiota, improves gut barrier function, and attenuates metabolic endotoxemia in rats with diet-induced obesity. Nutrients. 2016; 8(3), 126. doi:10.3390/nu8030126

75. Remier RA, Maurer AD, Eller LK, Hallam MC, Shaykhutdinov R, Vogel HJ, Weljie AM. Satiety hormone and metabolomic response to an intermittent high energy diet differs in rats consuming long-terms diets high in protein or prebiotic fiber. Journal of Proteome Research. 2012; 11(8), 4065-4074. doi:10.1021/pr300487s 